\title{
INDICADOR DE QUALIDADE: TAXA DE MORTALIDADE INTRAOPERATÓRIA E PÓS-OPERATÓRIA
}

\author{
Quality indicator: intraoperative and postoperative mortality rate
}

\section{Indicador de calidad: tasa de mortalidad intraperatoria y postoperatoria}

\author{
Jaqueline Lopes Gouveia ${ }^{1 *}$ (D), Priscila Buck de Oliveira Ruiz² (D), Dalva Maria da Silveira ${ }^{3}$ (D), Ângela Silveira Gagliardo Calil ${ }^{4}$ (D), \\ Renata Prado Bereta Vilela ${ }^{5}$ (D), Paula Buck de Oliveira Ruiz ${ }^{6}$ (D)
}

RESUMO: Objetivo: Identificar e caracterizar o indicador de qualidade de taxa de mortalidade intraoperatória e pós-operatória. Método: Estudo retrospectivo, quantitativo, com delineamento descritivo, transversal e correlação entre variáveis, realizado de janeiro a dezembro de 2017 em 18.337 prontuários. Foram utilizados métodos de estatística descritiva e inferencial, analisando-se questões de probabilidade de uma população com base nos dados da amostra. Resultados: No período estudado, houve 18.337 pacientes com taxa de mortalidade intraoperatória de 1,75\%, e a referente aos sete primeiros dias de pós-operatório foi de 1,76\%. Destes, 191 (58,95\%) eram do sexo masculino, 32,71\% dos pacientes foram classificados como American Society of Anesthesiologists III, e 80,24\% das cirurgias de caráter de urgência, classificadas como limpas, tiveram tempo médio de duração de até 120 minutos. Conclusão: A taxa de mortalidade encontrada na instituição está em conformidade com os valores descritos pelo Compromisso com a Qualidade Hospitalar. Houve correlação significativa entre as variáveis: tempo de cirurgia (até 120 minutos) e caráter de urgência; e classificação da cirurgia (limpa) e período de morte (até sete dias). Palavras-chave: Morte. Período intraoperatório. Período pós-operatório. Hospitais de ensino.

ABSTRACT: Objective: To identify and characterize the quality indicator of intraoperative and postoperative mortality rate. Method: This is a retrospective, quantitative, descriptive, cross-sectional study with variable correlation, based on 18,337 medical records and conducted from January to December 2017. The methods used were descriptive and inferential statistics, with analysis of probability issues of a population according to sample data. Results: In the study period, 18,337 patients underwent surgery, with a mortality rate of $1.75 \%$ in the intraoperative period and $1.76 \%$ in the first seven postoperative days. Among them, 191 (58.95\%) were men, and $32.71 \%$ were classified as American Society of Anesthesiologists class III; $80.24 \%$ of urgent surgeries, considered clean, had a mean duration of up to 120 minutes. Conclusion: The mortality rate found in the facility is in accordance with the values described by the Commitment to Hospital Quality. The following variables showed a significant correlation: operative time (up to 120 minutes) and urgent surgery; and surgical contamination (clean) and death period (up to seven days). Keywords: Death. Intraoperative period. Postoperative period. Hospitals, teaching.

RESUMEN: Objetivo: Identificar el indicador de calidad de las tasas de mortalidad intraoperatoria y posoperatoria. Método: Estudio retrospectivo, cuantitativo, con diseño descriptivo, transversal y correlación entre variables, realizado de enero a diciembre de 2017 en 18.337 registros. Se utilizaron métodos de estadística descriptiva e inferencial, analizando la probabilidad de una población a partir de los datos muestrales. Resultados: Durante el período de estudio, hubo 18.337 pacientes, con una tasa de mortalidad intraoperatoria del 1,75\%, y la de los primeros siete días postoperatorios del 1,76\%. De estos, 191 (58,95\%) eran hombres, 32,71\% de los pacientes fueron clasificados como American Society of Anesthesiologists III, 80,24\% de las cirugías urgentes, clasificadas como limpias, tuvieron una duración media de hasta 120 minutos. Conclusión: La tasa de mortalidad encontrada en la Institución está de acuerdo con los valores descritos por el Compromiso con la Calidad Hospitalaria. Hubo una correlación significativa entre las variables: tiempo de cirugía (hasta 120 minutos) y urgencia; clasificación de cirugía (limpia) y período de muerte (hasta 7 días). Palabras clave: Muerte. Periodo intraoperatorio. Periodo posoperatorio. Hospitales de enseñanza.

\footnotetext{
'Especialista em Centro Cirúrgico, Recuperação Anestésica e Central de Material e Esterilização pela Faculdade de Medicina de São José do Rio Preto (FAMERP). Enfermeira no Hospital de Base de São José do Rio Preto - São José do Rio Preto (SP), Brasil.

2Mestrandado Programade Pós-Graduação em Enfermagem daFaculdade de Medicinade São Josédo Rio Preto. Enfermeiracoordenadorado bloco operatório do Hospital de Base deSão José do Rio Preto-São José do Rio Preto (SP), Brasil. ${ }^{3}$ Doutora em Ciências da Saúde pela Faculdade de Medicina de São José do Rio Preto. Docente do curso de graduação de enfermagem da FAMERP - São José do Rio Preto (SP), Brasil.

‘Mestre em Ciências da Saúde pela FAMERP. Docente do curso de graduação de Enfermagem na FAMERP - São José do Rio Preto (SP), Brasil.

${ }_{5}^{5}$ Mestre em Enfermagem pela FAMERP. Docente do Programa de Integração Comunitária do curso de Medicina da FACERES - São José do Rio Preto (SP), Brasil.

${ }^{6}$ Doutoranda do Programa de Pós-Graduação em Gerenciamento em Enfermagem da Universidade de São Paulo - São Paulo (SP), Brasil.

*Autora correspondente: jaquelinelgouveia@hotmail.com

Recebido: 23/04/2020 - Aprovado: 26/10/2020

https://doi.org/10.5327/Z1414-4425202000040007
} 


\section{INTRODUÇÃo}

A taxa de mortalidade hospitalar vem sendo utilizada como um indicador de qualidade, buscando identificar os cuidados prestados em alguns procedimentos nos quais o óbito não é um acontecimento raro ${ }^{1}$. O óbito cirúrgico é definido como pacientes que morreram durante o procedimento anestésico-cirúrgico ou em até sete dias depois dele².

A segurança do paciente tem sido preocupação mundial desde o ano 2000, quando o Instituto de Medicina dos Estados Unidos divulgou o relatório nomeado To err is human: building a safer health care system, mostrando que aproximadamente $98 \mathrm{mil}$ pessoas morrem anualmente em decorrência de erros médi$\cos ^{3}$. No contexto cirúrgico, um desafio é o cuidado seguro. Estimativas demonstram que são realizadas 234 milhões de cirurgias, cuja incidência de óbitos é de dois a sete milhões, porém metade dessas mortes poderia ser evitada ${ }^{4}$.

Com o intuito de despertar a compreensão profissional e a obrigação política para garantir a segurança na assistência à saúde, a Aliança Mundial para a Segurança do Paciente iniciou a campanha Cirurgias Seguras Salvam Vidas, cujo propósito é promover a redução da taxa de morbimortalidade resultante de procedimentos cirúrgicos. Essa é a quarta meta mundial da Organização Mundial da Saúde, com o objetivo de aumentar a qualidade dos serviços de saúde. A campanha contempla quatro itens: infecções de sítio cirúrgico, anestesia segura, equipes cirúrgicas seguras e indicadores da assistência cirúrgica ${ }^{5}$.

Algumas organizações, com a ideia de orientar a assistência à saúde de qualidade e segurança, como a Joint Commission on Accreditation of Healthcare Organizations, apresentaram programas para proporcionar saúde ao paciente e diminuir as ocorrências de incidentes em ambientes de precaução ${ }^{6}$.

O Ministério da Saúde, por meio da Agência Nacional de Vigilância Sanitária, lançou no Brasil seis protocolos operacionais que abordam a relação entre terapia medicamentosa, risco de quedas, higienização das mãos, prevenção de lesões por pressão, identificação do paciente e segurança durante procedimentos cirúrgicos ${ }^{7}$. Julgando que a segurança do paciente é a diminuição, a um mínimo aceitável, do risco de um dano dispensável associado ao cuidado de saúde ${ }^{8}$, entende-se como de grande importância reconhecer os incidentes que ocorrem no centro cirúrgico (CC).

A qualidade da assistência, não apenas no CC, está ligada principalmente ao controle de não conformidades. Considera-se uma grave não conformidade o óbito cirúrgico do paciente nas primeiras 24 horas após o procedimento anestésico-cirúrgico ${ }^{9}$. Observa-se que o indicador de qualidade trata apenas do número absoluto de mortes do intraoperatório. Daí a importância do presente estudo de discutir a taxa de mortalidade cirúrgica como um indicador da assistência prestada no período perioperatório.

\section{OBJETIVO}

Identificar e caracterizar o indicador de qualidade de taxa de mortalidade intraoperatória e pós-operatória.

\section{MÉTODO}

Trata-se de estudo retrospectivo, quantitativo, com delineamento descritivo, transversal e correlação entre variáveis. A pesquisa foi desenvolvida em um hospital de ensino de capacidade extra (708 leitos) localizado na Região Sudeste do Brasil, em São José do Rio Preto, estado de São Paulo. A instituição presta atendimento a dois milhões de habitantes de 102 municípios da Divisão Regional de Saúde de Rio Preto, que são pacientes dos sistemas público e privado de saúde. O CC é composto de 25 salas operatórias, abrangendo, em média, 18.337 pacientes submetidos a cirurgias/ano, dos quais $10.642(58,03 \%)$ são atendidos pelo Sistema Único de Saúde e 7.695 (41,96\%) pelo Sistema de Saúde Suplementar.

A coleta de dados foi realizada no CC entre julho e agosto de 2018, sendo incluídos pacientes submetidos a cirurgias eletivas, de urgência e emergência e que morreram no período intraoperatório e em até sete dias após o procedimento anestésico-cirúrgico de janeiro a dezembro de 2017. Os critérios de exclusão compreenderam pacientes menores de 14 anos, que foram submetidos à cirurgia na sala de pequenos procedimentos, a cirurgias oftalmológicas, obstétricas, e no hospital interno infantil, que faz parte do complexo da instituição e da captação de órgãos American Society of Anesthesiologists (ASA) VI.

Para levantamento das informações, foi utilizada a base de dados do hospital, com dados fornecidos pelos gestores dos setores de faturamento e CC. Para tal coleta, realizou-se uma análise dos prontuários (online) de 18.337 pacientes operados entre $1^{\circ}$ de janeiro e 31 de dezembro de 2017 - 324 registros eram de pacientes que vieram a óbito no período intraoperatório ou em até sete dias após o procedimento. Os pacientes 
que morreram foram caracterizados quanto a: sexo, idade, especialidade médica, classificação de tipo de cirurgia (eletiva e urgência), classificação da cirurgia segundo potencial de contaminação (limpa, contaminada, potencialmente contaminada e infectada), tempo cirúrgico (porte cirúrgico) e classificação do estado físico de acordo com a ASA.

Para o cálculo da taxa de mortalidade cirúrgica, usou-se a fórmula Compromisso com a Qualidade Hospitalar $(\mathrm{CQH})$, definida pela razão entre o número de óbitos operatórios dividido pelo número de cirurgias realizadas multiplicado por $100^{2}$.

Foram utilizados métodos de estatística descritiva e de estatística inferencial, analisando-se questões de probabilidade de uma população com base nos dados da amostra. Em alguns momentos, dada a necessidade e para melhor entendimento, empregaram-se os seguintes métodos: média, mediana, teste de Kolmogorov-Smirnov, regressão de Poisson, teste de Mann-Whitney, correlação de Spearman, significância e erro padrão. Os cruzamentos realizados foram: tipo de agendamento e sexo; tipo de agendamento e especialidade médica; tipo de agendamento e idade; tipo de agendamento e classificação da cirurgia; tipo de agendamento e ASA; tipo de agendamento e tempo cirúrgico (em minutos); óbito após cirurgia (dias) e natureza da cirurgia; ASA e natureza da cirurgia; ASA e idade; e ASA e sexo.

As variáveis passaram por análise estatística inferencial, na qual o método de análise se dá por meio do resultado do teste de normalidade de variável. Os métodos escolhidos para abordagem das análises de variação dos resultados entre os grupos analisados visam, em suma, verificar a relação entre eles, com parametrização de uma das variáveis como dependente e a outra como independente, objetivando a análise de predição entre ambas. Os resultados foram obtidos por $\mathrm{p}<0,05$, caracterizando significância entre os grupos estudados. Todos os testes contemplam erro alfa de $5 \%$ e confiabilidade de $95 \%$.

Os dados foram coletados pela primeira pesquisadora, após aprovação do Comitê de Ética em Pesquisa da instituição sede do estudo, sob parecer $\mathrm{n}^{\circ}$ 2.775.232.

\section{RESULTADOS}

No período estudado (entre janeiro e dezembro de 2017), foram realizados procedimentos anestésico-cirúrgicos em 18.337 pacientes, variando de $1.740(9,48 \%)$, em agosto, a 1.389 (7,57\%), em abril. O indicador de óbito cirúrgico apresentou

taxa de $1,76 \%$ ao ano, variando de 2,25 a $1,15 \%$, e mediana de $1,75 \%$, conforme se pode verificar na Tabela 1 .

Em relação à caracterização da amostra (Tabela 2), houve predomínio de pacientes do sexo masculino $(58,95 \%)$ e de cirurgias de urgência $(59,23 \%)$. A faixa etária mais representativa foi de 61 a 80 anos $(45,37 \%)$, sendo nesse caso as cirurgias de urgência $(46,15 \%)$ as mais prevalentes, e a menor incidência contempla pacientes com idade de até 20 anos $(0,93 \%)$.

Quanto ao perfil traçado pelas especialidades médicas, foram encontrados maiores números em procedimentos da equipe de cirurgia geral (50\%), com predomínio nas urgências $(54,23 \%)$, e menores números na endoscopia $(0,62 \%)$, em eletivas $(3,13 \%)$.

No que se refere à classificação das cirurgias, houve predomínio na amostra de procedimentos limpos, totalizando $46,91 \%$. Destes, as cirurgias de urgência foram as mais presentes (43,08\%). As cirurgias potencialmente contaminadas representaram $27,47 \%$ dos procedimentos realizados, e, destes, as cirurgias de urgência também foram as mais expressivas $(28,85 \%)$.

Acerca da classificação ASA, pode-se observar na Tabela 3 que $32,71 \%$ dos pacientes que morreram em até sete dias apresentavam a classificação ASA III e haviam realizado cirurgias eletivas, seguidos de $24,38 \%$ com ASA IV, que realizaram cirurgias de urgência. Houve relação significativa para esse cruzamento, com $\mathrm{p}=0,036$.

Tabela 1. Cirurgias realizadas, óbitos cirúrgicos e taxa de mortalidade cirúrgica, no período de janeiro a dezembro de 2017.

\begin{tabular}{|l|c|c|c|}
\hline \multirow{2}{*}{ Mês } & $\begin{array}{c}\text { Cirurgias } \\
\text { realizadas }\end{array}$ & $\begin{array}{c}\text { Óbito } \\
\text { cirúrgico }\end{array}$ & $\begin{array}{c}\text { Taxa de } \\
\text { mortalidade } \\
\text { cirúrgica }\end{array}$ \\
\cline { 2 - 4 } & $\mathbf{N}$ & $\mathbf{N}$ & $\%$ \\
\hline Janeiro & 1.614 & 26 & 1,61 \\
\hline Fevereiro & 1.441 & 30 & 2,08 \\
\hline Março & 1.544 & 26 & 1,68 \\
\hline Abril & 1.389 & 25 & 1,80 \\
\hline Maio & 1.593 & 36 & 2,25 \\
\hline Junho & 1.517 & 18 & 1,18 \\
\hline Julho & 1.702 & 29 & 1,70 \\
\hline Agosto & 1.740 & 20 & 1,15 \\
\hline Setembro & 1.548 & 31 & 2,00 \\
\hline Outubro & 1.422 & 25 & 1,75 \\
\hline Novembro & 1.422 & 28 & 1,96 \\
\hline Dezembro & 1.405 & 30 & 2,13 \\
\hline TOTAL & 18.337 & 324 & 1,76 \\
\hline
\end{tabular}


Tabela 2. Caracterização dos pacientes no período intraoperatório e sete dias após o procedimento, segundo tipo de procedimento cirúrgico.

\begin{tabular}{|c|c|c|c|c|c|c|}
\hline \multirow{2}{*}{ Sexo } & \multicolumn{2}{|c|}{ Eletiva } & \multicolumn{2}{|c|}{ Urgência } & \multicolumn{2}{|c|}{ Total } \\
\hline & $\mathbf{N}$ & $\%$ & $\mathbf{N}$ & $\%$ & $\mathbf{N}$ & $\%$ \\
\hline Masculino & 37 & 57,81 & 154 & 59,23 & 191 & 58,95 \\
\hline Feminino & 27 & 42,19 & 102 & 39,23 & 129 & 39,81 \\
\hline Sem informação & 0 & 0 & 4 & 1,54 & 4 & 1,23 \\
\hline Total & 64 & 100 & 260 & 100 & 324 & 100 \\
\hline \multicolumn{7}{|l|}{ Idade (anos) } \\
\hline Até 20 & 1 & 1,56 & 2 & 0,77 & 3 & 0,93 \\
\hline 21 a 40 & 3 & 4,69 & 26 & 10 & 29 & 8,95 \\
\hline 41 a 60 & 24 & 37,50 & 70 & 26,92 & 94 & 29,01 \\
\hline 61 a 80 & 27 & 42,19 & 120 & 46,15 & 147 & 45,37 \\
\hline$>80$ & 9 & 14,06 & 42 & 16,15 & 51 & 15,74 \\
\hline Total & 64 & 100 & 260 & 100 & 324 & 100 \\
\hline \multicolumn{7}{|l|}{ Especialidade } \\
\hline Cirurgia geral & 21 & 32,81 & 141 & 54,23 & 162 & 50 \\
\hline Neurocirurgia & 13 & 20,31 & 53 & 20,38 & 66 & 20,37 \\
\hline Cirurgia vascular & 4 & 6,25 & 19 & 7,31 & 23 & 7,10 \\
\hline Ortopedia / traumatologia & 8 & 12,50 & 12 & 4,62 & 20 & 6,17 \\
\hline Cirurgia cardiovascular & 10 & 15,63 & 7 & 2,69 & 17 & 5,25 \\
\hline Urologia & 2 & 3,13 & 10 & 3,85 & 12 & 3,70 \\
\hline Cirurgia torácica & 3 & 4,69 & 7 & 2,69 & 10 & 3,09 \\
\hline Proctologia & 0 & 0,00 & 7 & 2,69 & 7 & 2,16 \\
\hline Otorrinolaringologia & 1 & 1,56 & 4 & 1,54 & 5 & 1,54 \\
\hline Endoscopia & 2 & 3,13 & 0 & 0 & 2 & 0,62 \\
\hline Total & 64 & 100 & 260 & 100 & 324 & 100 \\
\hline \multicolumn{7}{|l|}{ Classificação } \\
\hline Limpa & 40 & 62,50 & 112 & 43,08 & 152 & 46,91 \\
\hline Potencialmente Contaminada & 14 & 21,88 & 75 & 28,85 & 89 & 27,47 \\
\hline Contaminada & 8 & 12,50 & 57 & 21,92 & 65 & 20,06 \\
\hline Infectada & 1 & 1,56 & 15 & 5,77 & 16 & 4,94 \\
\hline Sem informação & 1 & 1,56 & 1 & 0,38 & 2 & 0,62 \\
\hline Total & 64 & 100 & 260 & 100 & 324 & 100 \\
\hline
\end{tabular}

Tabela 3. Distribuição da American Society of Anesthesiology (ASA) e do tempo cirúrgico, de acordo com o tipo de agendamento.

\begin{tabular}{|c|c|c|c|c|c|c|}
\hline \multirow{2}{*}{$\begin{array}{l}\text { Informação } \\
\text { ASA }\end{array}$} & \multicolumn{2}{|c|}{ Eletiva } & \multicolumn{2}{|c|}{ Urgência } & \multicolumn{2}{|c|}{ Total } \\
\hline & $\mathbf{N}$ & $\%$ & $\mathbf{N}$ & $\%$ & $\mathbf{N}$ & $\%$ \\
\hline ASA I & 2 & 3,13 & 6 & 2,31 & 8 & 2,47 \\
\hline ASA II & 21 & 32,81 & 43 & 16,54 & 64 & 19,75 \\
\hline ASA III & 22 & 34,37 & 84 & 32,30 & 106 & 32,71 \\
\hline ASA IV & 14 & 21,87 & 65 & 25 & 79 & 24,38 \\
\hline ASA V & 3 & 4,68 & 12 & 4,61 & 15 & 4,62 \\
\hline Sem informação & 2 & 3,13 & 50 & 19,23 & 51 & 15,74 \\
\hline Total & 64 & 100 & 260 & 100 & 324 & 100 \\
\hline \multicolumn{7}{|c|}{ Tempo cirúrgico (minutos) } \\
\hline Até 120 & 14 & 21,88 & 128 & 49,23 & 142 & 43,83 \\
\hline 121 a 240 & 24 & 37,50 & 97 & 37,31 & 121 & 37,35 \\
\hline 241 a 360 & 16 & 25 & 24 & 9,23 & 40 & 12,35 \\
\hline 361 a 480 & 7 & 10,94 & 10 & 3,85 & 17 & 5,25 \\
\hline$>480$ & 3 & 4,69 & 1 & 0,38 & 4 & 1,23 \\
\hline Total & 64 & 100 & 260 & 100 & 324 & 100 \\
\hline
\end{tabular}


Tabela 4. Distribuição de óbitos cirúrgicos de pacientes, de acordo com os dias e a classificação da cirurgia.

\begin{tabular}{|c|c|c|c|c|c|c|c|c|c|c|c|c|}
\hline \multirow[t]{2}{*}{ Dias } & \multicolumn{2}{|c|}{ Contaminada } & \multicolumn{2}{|c|}{ Infectada } & \multicolumn{2}{|c|}{ Limpa } & \multicolumn{2}{|c|}{$\begin{array}{l}\text { Potencialmente } \\
\text { contaminada }\end{array}$} & \multicolumn{2}{|c|}{ Sem informação } & \multicolumn{2}{|c|}{ Total } \\
\hline & $\mathbf{N}$ & $\%$ & $\mathbf{N}$ & $\%$ & $\mathbf{N}$ & $\%$ & $\mathbf{N}$ & $\%$ & $\mathbf{N}$ & $\%$ & $\mathbf{N}$ & $\%$ \\
\hline 0 & 11 & 16,92 & 2 & 12,50 & 39 & 25,66 & 13 & 14,61 & 0 & 0 & 65 & 20,06 \\
\hline 1 & 28 & 43,08 & 4 & 25,00 & 25 & 16,45 & 18 & 20,22 & 2 & 100 & 77 & 23,77 \\
\hline 2 & 6 & 9,23 & 5 & 31,25 & 20 & 13,16 & 21 & 23,60 & 0 & 0 & 52 & 16,05 \\
\hline 3 & 7 & 10,77 & 1 & 6,25 & 20 & 13,16 & 12 & 13,48 & 0 & 0 & 40 & 12,35 \\
\hline 4 & 5 & 7,69 & 1 & 6,25 & 13 & 8,55 & 12 & 13,48 & 0 & 0 & 31 & 9,57 \\
\hline 5 & 1 & 1,54 & 2 & 12,50 & 12 & 7,89 & 4 & 4,49 & 0 & 0 & 19 & 5,86 \\
\hline 6 & 2 & 3,08 & 1 & 6,25 & 16 & 10,53 & 6 & 6,74 & 0 & 0 & 25 & 7,72 \\
\hline 7 & 5 & 7,69 & 0 & 0 & 7 & 4,61 & 3 & 3,37 & 0 & 0 & 15 & 4,63 \\
\hline Total & 65 & 100 & 16 & 100 & 152 & 100 & 89 & 100 & 2 & 100 & 324 & 100 \\
\hline
\end{tabular}

Foi demonstrado que $43,83 \%$ dos pacientes morreram em procedimentos realizados em até 120 minutos. Nesse período de procedimento, a cirurgia de urgência foi a mais representativa $(49,23 \%)$. Na sequência, o período de 121 a 240 minutos apresentou $37,35 \%$ de óbitos, entre os quais as cirurgias de urgência também foram as mais expressivas (37,31\%). Tratando-se de correlação, constatou-se valor significativo no cruzamento dessas variáveis, $\operatorname{com} \mathrm{p}=0,000$.

Quanto à classificação das cirurgias e ao período de óbito, conforme descrito na Tabela 4, houve variação de mortes de 15 no sétimo dia a 77 no primeiro dia. A maior porcentagem de óbitos foi nas cirurgias limpas (152 pacientes / 46,9\%). Ao analisar o dia com a classificação da cirurgia, foram mais representativas as cirurgias contaminadas com óbito no primeiro dia (28 pacientes). Ao realizar análise referente à correlação das variáveis, houve associação significativa, $\operatorname{com} p=0,040$.

\section{DISCUSSÃO}

O presente estudo demonstra o indicador de taxa de mortalidade operatória com mediana de 1,75\%. Observa-se, em relação ao mesmo indicador, que no Programa $\mathrm{CQH}$, no ano de 2017, para 49 hospitais gerais notificantes, a mediana foi de $0,12 \%$, com variação de 0,05 a $4,96 \%^{2}$.

Um estudo realizado no complexo hospitalar Hospital das Clínicas da Faculdade de Medicina da Universidade de São Paulo (HCFMUSP), no período de 2007 a 2011, mostrou que a taxa de mortalidade cirúrgica variou de 1 a $2,3 \%{ }^{10}$. Estudo do tipo caso controle desenvolvido em hospital público em Santa Catarina apresentou taxa de $9,8 \%{ }^{11}$.
Em outra pesquisa em que foi feita revisão sistemática da literatura, os autores demonstraram que as taxas de mortalidade operatória são maiores no Brasil e em outros países em desenvolvimento, quando comparadas a de países desenvolvidos ${ }^{12}$.

Um estudo de coorte prospectivo com 187 pacientes, realizado em Porto Alegre, encontrou que $48 \%$ dos pacientes que morreram eram do sexo masculino ${ }^{13}$. Os dados estão em consonância com o trabalho atual, que observou predomínio do sexo masculino (58,95\%). Já em dois estudos relacionados a óbitos em pacientes submetidos a cirurgias ortopédicas, viu-se o predomínio do sexo feminino com $76,9^{14}$ e $72,3 \%{ }^{15}$, opondo-se ao resultado do estudo atual.

Um estudo realizado em um banco de dados de hospitais franceses, em 2010, que verificou a derivação e validação do Escore Pré-Operatório para Predizer Mortalidade PósOperatória, mostrou que o risco de mortalidade hospitalar após cirurgias ortopédicas de grande porte foi de 3,46\%, enquanto para cirurgias vasculares foi de $1,09 \%{ }^{16}$. Os dados supracitados divergem do estudo atual, haja vista que as maiores taxas encontradas foram em cirurgias gerais (50\%) e nas neurocirurgias (20,37\%). Nesse sentido, uma pesquisa em um hospital público de grande porte do Rio Grande do Sul, em 2017, apresentou dados semelhantes aos encontrados aqui, em que a causa de óbitos mais frequente foi a neurocirurgia $(44,3 \%)$, seguida da cirurgia geral $(33,3 \%)^{17}$.

Tratando-se da faixa etária, o óbito cirúrgico foi predominante em idosos de 61 a 80 anos, porém não foi evidenciada correlação entre as variáveis idade e óbito ocasionado pelas cirurgias. Estudo feito em um hospital público do interior de São Paulo referente ao óbito cirúrgico demonstrou que foi 
prevalente a idade de 72 a 79 anos $^{18}$, no entanto outro estudo indicou que a idade em si não foi fator de risco independente ${ }^{19}$, sustentando os resultados apresentados nesse estudo.

É possível comprovar, com base em uma revisão sistemática da literatura, que a maioria dos óbitos perioperatórios ocorre em pacientes classificados como ASA igual ou superior a III e em cirurgias de urgência.

Uma pesquisa no HCFMUSP em que os autores identificaram a taxa de mortalidade verificou que a alta complexidade da população atendida nessa instituição foi de 57,09\%, também na mesma classificação de ASA $\geq \operatorname{III}^{11}$. Os dados supracitados corroboram os resultados do estudo atual, que mostrou nível de significância para tais variáveis. Estudo realizado durante três anos (de 2008 a 2010) em um hospital de ensino em Edmonton, no Canadá, demonstrou que a maior parte dos pacientes com 80 anos ou mais submetidos a procedimentos cirúrgicos com a equipe da cirurgia geral de emergência tinha limitações funcionais, com prevalência de pacientes com classificação ASA III. Além disso, mais de $60 \%$ deles apresentaram boa sobrevida em longo prazo ${ }^{20}$.

Investigação recente mostra que a duração cirúrgica superior a 130 minutos em cirurgias eletivas é fator de risco independente para complicações, bem como o tempo de internação ${ }^{21}$. Sendo assim, os dados mencionados reafirmam o que foi encontrado no estudo atual, estando, assim, estatisticamente correlacionados o tipo de procedimento cirúrgico e o tempo cirúrgico. Vale ressaltar a importância de acompanhamento aos médicos residentes por médicos preceptores, item destacado na literatura pelo New South Wales Health Emergency Surgery Guidelines, como um dos principais objetivos de replanejamento dos serviços, pois, dessa forma, o tempo cirúrgico será menor, diminuindo uma variável impactante na taxa de mortalidade cirúrgica ${ }^{21}$.

Estudo realizado em oito hospitais de vários países (Canadá, Índia, Jordânia, Filipinas, Nova Zelândia, Tanzânia, Inglaterra e Estados Unidos) verificou a necessidade e o acompanhamento de todos os pacientes, em todas as etapas pelas quais eles passam no CC. Observou-se que todos os índices analisados decaíram de valor, inclusive a taxa de mortalidade, de 1,5 para $0,8 \%$, representando redução de $55 \%$ na taxa de mortalidade pós-operatória ${ }^{22}$.

No estudo atual, foi possível apontar a maior taxa de mortalidade decorrente de cirurgias limpas em até 24 horas, mostrando relação estatística, entretanto um estudo prospectivo com o objetivo de comparar os resultados clínicos antes e depois da implantação do Projeto ACERTO ${ }^{23}$, que é um conjunto de rotinas com o intuito de acelerar a recuperação de pacientes submetidos a operações abdominais, comprovou a prevalência de mortalidade em cirurgias classificadas como potencialmente contaminadas, mas não houve significância estatística. Tais resultados não corroboram os obtidos na presente pesquisa.

Este estudo apresenta limitações, como a escassez de estudos nacionais e internacionais atualizados relacionados ao tema em questão e a apresentação de dados associados com a mortalidade e a incidência de reoperações. A melhoria dos desfechos depende fundamentalmente de alguns fatores, como: o reconhecimento do paciente quanto à sua classificação ASA, que considera o estado físico do indivíduo; a busca da redução do tempo cirúrgico, que vai possibilitar que o paciente fique menos exposto/suscetível a complicações; e o seguimento de rotinas e protocolos que propiciem o melhor cuidado ao paciente cirúrgico, independentemente de sua classificação segundo o potencial de contaminação. O presente estudo evidenciou elevada taxa de mortalidade em cirurgias classificadas como limpas, o que diverge de pesquisas nacionais e internacionais.

Oferecer cuidado seguro ao paciente em todas as etapas cirúrgicas é um desafio para todas as instituições prestadoras de serviços de assistência à saúde, com o intuito de oferecer redução da morbimortalidade decorrente dos procedimentos anestésico-cirúrgicos ${ }^{4}$.

\section{CONCLUSÃO}

A análise das informações de 18.337 pacientes permitiu calcular a taxa de mortalidade operatória, no valor de 1,75\% na instituição estudada, incidência que está entre os valores descritos pelo $\mathrm{CQH}$. Os achados evidenciaram, em relação ao perfil dos pacientes, predomínio do sexo masculino, idades entre 61 e 80 anos, classificados como ASA III, em cirurgias de caráter de urgência, especialidade médica da cirurgia geral, em cirurgias classificadas como limpas e tempo médio de até 120 minutos.

O tema abordado no estudo é de grande relevância, tendo em vista a escassez de literatura a respeito dele. Esta pesquisa possibilitou o mapeamento da taxa e da caracterização de mortalidade operatória. Os dados contribuem para o diagnóstico situacional e para a elaboração de estratégias que propiciem reduzir essa taxa na população-alvo, melhorando a qualidade da assistência prestada pela equipe de enfermagem e pela equipe perioperatória como um todo. 


\section{REFERÊNCIAS}

1. Noronha JC, Martins M, Travassos C, Campos MR, Maia P, Panezzuti R. Mortalidade hospitalar após cirurgia de revascularização do miocárdio. Cad Saúde Pública. 2004;20(Supl. 2):S322-30. http://doi. org/10.1590/S0102-311X2004000800025

2. Duarte IG, Nagai MH, Mota NVVP, Bittar OJNV, Nishikuni YY. Compromisso com a qualidade hospitalar. $3^{\circ}$ Caderno de Indicadores CQH. São Paulo: Van Moorsel; 2009.

3. Kohn LT, Corrigan JM, Donaldson MS. To err is human: building a safer health system. Washington, D.C.: National Academy Press; 2000.

4. Bezerra WR, Bezerra ALQ, Paranaguá TTB, Bernardes MJC, Teixeira C. Ocorrência de incidentes em um centro cirúrgico: estudo documental. Rev Eletr Enf. 2015;17(4). https://doi.org/10.5216/ree.v17i4.33339

5. Organização Pan-Americana da Saúde. Cirurgias seguras salvam vidas. Segundo desafio global para a segurança do paciente. Rio de Janeiro: Organização Pan-Americana da Saúde; Ministério da Saúde; Agência Nacional de Vigilância Sanitária. Brasília; 2009 [acesso em 11 maio 2018]. Disponível em: http://bvsms.saude.gov.br/bvs/publicacoes/ seguranca_paciente_cirurgias_seguras_salvam_vidas.pdf

6. Joint Commission International. The Joint Commission announces 2014 national patient safety goal. Joint Commission Perspec. 2013;33(7):1-20.

7. Agência Nacional de Vigilância Sanitária (ANVISA). Segurança do paciente. [Internet]. Brasília: Ministério da Saúde; 2014 [acesso em 11 maio 2018]. Disponível em: http://www20.anvisa.gov.br/ segurancadopaciente/index.php/publicacoes/category/div ersos

8. World Health Organization (WHO). World alliance for patient safety. Taxonomy. The conceptual framework for the international classification for patient safety [Internet]. Genebra: World Health Organization; 2009 [acesso em 11 maio 2018]. Disponível em: http://www.who. int/patientsafety/taxonomy/icps_full_report.pdf

9. Grazziano ES, Viana DL, Harada MJCS, Luz M. Enfermagem perioperatória e cirurgia segura. São Paulo: Yendis; 2016. 344 p.

10. São Paulo. Governo do Estado de São Paulo. Relatório de Gestão HCFMUSP. Hospital das Clínicas da Faculdade de Medicina da Universidade de São Paulo. São Paulo: Governo do Estado de São Paulo; 2010.

11. Leandro GS, Parolim SC, Moro C, Carvalho DR. Mineração de dados na avaliação de óbitos após cirurgia de amputação. J Vasc Bras. 2018;17(1):10-8. http://doi.org/10.1590/1677-5449.008317

12. Braz LG, Braz DG, Cruz DS, Fernandes LA, Módolo NSP, Braz JRC. Mortality in anesthesia: a systematic review. Clinics. 2009;64(10):9991006. https://doi.org/10.1590/S1807-59322009001000011
13. Stahlschmidt A, Novelo B, Freitas LA, Passos SC, Dussán-Sarria JA, Félix EA, et al. Preditores de mortalidade intra-hospitalar em pacientes submetidos a cirurgias não eletivas em um hospital universitário: uma coorte prospectiva. Braz J Anesthesiol. 2018;68(5):492-8. https:// doi.org/10.1016/j.bjane.2018.04.009

14. Barbosa TA, Souza AMF, Leme FCO, Grassi LDV, Cintra FB, Lima $\mathrm{RM}$, et al. Complicações perioperatórias e mortalidade em pacientes idosos submetidos a cirurgia para correção de fratura de fêmur: estudo prospectivo observacional. Braz J Anesthesiol. 2019;69(6):569-79. https://doi.org/10.1016/j.bjan.2019.09.004

15. Guerra MTE, Viana RD, Feil L, Feron ET, Maboni J, Vargas ASG. Mortalidade em um ano de pacientes idosos com fratura do quadril tratados cirurgicamente num hospital do Sul do Brasil. Rev Bras Ortop. 2017;52(1):17-23. https://doi.org/10.1016/j.rboe.2016.11.006

16. Manach LY, Collins G, Rodseth R, Bihan-Benjamin C, Biccard B, Riou B, et al. Preoperative Score to Predict Postoperative Mortality (POSPOM): derivation and validation. Anesthesiology. 2016;124(3):570-9. https:// doi.org/10.1097/ALN.0000000000000972

17. Machado LVL, Jardim DP. Óbito do paciente intensivo na recuperação pós-anestésica: uma experiência descontextualizada. Rev SOBECC. 2018;23(3):130-5. https://doi.org/10.5327/Z1414-4425201800030004

18. Moreira PS, Alcântara LRS, Matilde JD, Rinaldi LC, Avila MAG. Cirurgias realizadas em idosos em um hospital público do interior de São Paulo. Rev SOBECC. 2019;24(2):69-75. https://doi.org/10.5327/ Z1414-4425201900020004

19. Kawashima Y, Seo N, Morita K, Irita K, Iwao Y, Tsuzaki K, et al. Anaesthesia related mortality and morbidity in Japan (1999). J Anesth. 2002;16:319-31. https://doi.org/10.1007/s005400200049

20. Merani S, Payne J, Padwal RS, Hudson D, Widder SL, Khadaroo RG. Predictors of in-hospital mortality and complications in very elderly patients undergoing emergency surgery. World J Emerg Surg. 2014;9:43. https://doi.org/10.1186\%2F1749-7922-9-43

21. Stahlschmidt A, Novelo B, Freitas LA, Passos SC, Dussán-Sarria JA, Félix EA, et al. Preditores de mortalidade intra-hospitalar em pacientes submetidos a cirurgias não eletivas em um hospital universitário: uma coorte prospectiva. Rev Bras Anestesiol. 2018;68(5):492-8. https://doi.org/10.1016/j.bjane.2018.04.009

22. Mendelssonh P. Cirurgia segura: armadilhas na prática cirúrgica. Brasília Med. 2012;49(1):59-65.

23. Bicudo-Salomão A, Meireles MB, Caporossi C, Crotti PLR, AguilarNascimento JE. Impacto do projeto acerto na morbi-mortalidade pós-operatória em um hospital universitário. Rev Col Bras Cir. 2011;38(1):3-10. https://doi.org/10.1590/S0100-69912011000100002 